\title{
Gradient index lenses for flexural waves based on thickness variations
}

\author{
Alfonso Climente, Daniel Torrent, ${ }^{a)}$ and José Sánchez-Dehesa ${ }^{\text {b) }}$ \\ Wave Phenomena Group, Universitat Politècnica de València, C/Camino de vera s.n. (edificio 7F), \\ ES-46022 Valencia, Spain
}

(Received 11 July 2014; accepted 4 August 2014; published online 13 August 2014)

\begin{abstract}
This work presents a method for the realization of gradient index devices for flexural waves in thin plates. Unlike recent approaches based on phononic crystals, the present approach is based on the thickness-dependence of the dispersion relation of flexural waves, which is used to create gradient index devices by means of local variations of the plate's thickness. Numerical simulations of known circularly symmetrical gradient index lenses have been performed. These simulations have been done using the multilayer multiple scattering method and the results prove their broadband efficiency and omnidirectional properties. Finally, finite element simulations employing the full three-dimensional elasticity equations also support the validity of the designed approach. (C) 2014 AIP Publishing LLC. [http://dx.doi.org/10.1063/1.4893153]
\end{abstract}

The propagation of flexural waves in thin plates through periodic arrangements of scatterers or inclusions has been a hot topic in the last years. The scattering by holes in plates has been analyzed ${ }^{1-5}$ and the resulting band structures has been obtained. ${ }^{6-8}$ In addition, the control of the propagation of waves by means of inhomogeneous media, also named "gradient index" (GRIN) devices, has received a lot of attention due to the possibility of designing positive $\mathrm{e}^{9,10}$ and negative ${ }^{11-13}$ refraction devices. GRIN devices consist of a space region where the physical parameters defining the wave velocity are position-dependent, thus enforcing the wavefront to follow curved trajectories. These lenses have proved to be useful for structural testing. ${ }^{14,15}$

The local-dependence of the physical properties of the medium can be realized by several ways, but the most used for both acoustic and elastic waves is based on phononic crystals. In the long-wavelength limit, these composite can be characterized by some effective parameters. Varying locally the parameters of the unit cell, it is possible to change the effective speed of sound. ${ }^{16-18}$ In the case of elastic waves in thin plates, the simplest form of a phononic crystal-based device consist in creating an array of holes whose radii is changed according to a specific relationship hole radiusrefractive index.

Although the frequency response of the refractive devices described above is known to be broadband, the major drawback is that a GRIN device requires a continuous variation of the refractive index, which obviously cannot be done with discrete lattices of holes, since the result will always be a stepped profile. In practice, the holes must be done small enough to make this stepped profile as smooth as possible, but as the frequency of the propagating wave is increased, the precision requirements become unaffordable. ${ }^{19}$

However, the local-dependence of the elastic properties can also be obtained by means of thickness variations. This approach has been employed by Krylov and Tillman in

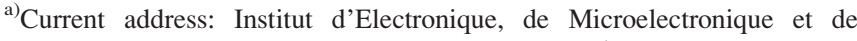
Nanotechnologie, UMR CNRS 8520, Université de Lille 1, 59655 Villeneuve dAscq, France.

b)jsdehesa@upv.es
}

$2004 .^{20}$ It is based on the peculiar dispersion relationship of flexural waves, which depends not only on the material of the plate but also on its thickness, which is a parameter that can be easily controlled at almost every scale. The approach has been extensively applied to carry out experimental investigations to achieve efficient damping of flexural waves; first, at the edge of plates ${ }^{21}$ and then at the center of plates ${ }^{22,23}$ which consists of designed pits with a layer of absorbing materials attached in the middle. Following this approach, ${ }^{24}$ the present authors also designed an insulating device for flexural waves.

In this Letter, we present a simple manner of creating GRIN lenses for flexural waves in thin plates based on the local variation of the plate's thickness. The tested devices consist of five circularly symmetric GRIN devices well known in optics (see, e.g., Ref. 25), whose index profiles are implemented in terms of thickness variations. Multiple scattering simulations of the lenses are also presented based on the solution of the two-dimensional flexural wave equation, and it is shown that the behavior is in agreement with the optical ray equation. Finally, a comparison of the simulation method with the solution obtained with the three-dimensional (3D) full elastic wave equation, using a commercial finite element simulator is presented showing very good agreement with our prediction.

Fig. 1 shows a schematic view of the gradient index device analyzed in the present work. The device is defined by a

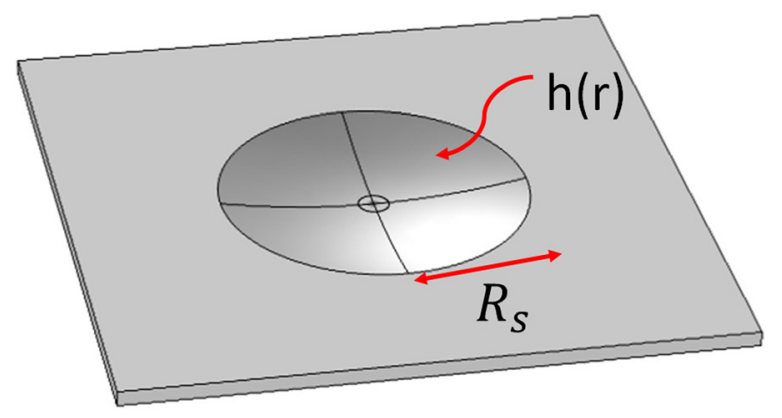

FIG. 1. Scheme of a circular flexural lens with radius $R_{s}$ with a radial dependent thickness $h(r)$ to achieve the desired refractive index $n(r)$. 
circular region $r \leq R_{s}$, where the thickness $h(r)$ of the plate is a function of the radial coordinate. This gradual variation of the plate's thickness can be modeled as a gradual variation of the refractive index, as will be shown below.

The equation of motion describing flexural waves in thin plates is modeled using the Kirchhoff-Love approximation in which the vertical displacement $W$ of the plate is obtained from the Timoshenko plate equation (assuming monochromatic time dependence of frequency $\omega$ )

$$
D \nabla^{4} W-\omega^{2} \rho h W=0,
$$

being $D=E h^{3} / 12\left(1-\nu^{2}\right)$ the flexural rigidity, $\rho$ the mass density, $h$ the thickness of the plate, $E$ the Young modulus, and $\nu$ the Poisson ratio. For plane wave propagation with wave number $k$, the above equation gives a quadratic dispersion relation

$$
k^{4}=\frac{\rho h \omega^{2}}{D},
$$

where it is seen that both the phase and group velocities of the waves are function of the plate's thickness $h$. It is straightforward to obtain the refractive index as a function of the position-dependent $h$ as ${ }^{24}$

$$
n(r, \theta)=\sqrt{\frac{h_{b}}{h(r, \theta)}},
$$

where $h_{b}$ is the background's thickness and $h(r, \theta)$ is the variation of the thickness in polar coordinates. It has been assumed that all the other elastic properties of the plate remain unchanged. Equation (3) describes how the refractive index increases with decreasing thickness. It is important to notice that, despite being an intrinsically dispersive medium, the refractive index does not depend on the frequency of the wave.

To test this approach, a set of refractive index lenses with circular symmetry has been studied. Table I shows the list of these lenses, corresponding to several solutions of the Luneburg problem with their respective variation of the refractive index. ${ }^{25}$ The last device corresponds to the elastic analogue of the optical concentrator previously described for electromagnetic waves. ${ }^{26}$ Note that all these lenses have been defined for optical waves, however, since they are obtained using the geometrical optics approximation, we assume that the solutions are also valid for flexural waves. This assumption has been applied previously for acoustics

TABLE I. Normalized refractive index of several solutions of the Luneburg problem (first four lenses). Additionally, a Concentrator lens (last lens) obtained by mapping the electromagnetic regime in the elastic one.

\begin{tabular}{lc}
\hline \hline Lens name & Refractive index (n) \\
\hline Luneburg & $n=\sqrt{2-r^{2}}$ \\
Maxwell Fish-Eye & $n=2 /\left(1+r^{2}\right)$ \\
$90^{\circ}$ rotating & $r n^{4}-2 n+r=0$ \\
Eaton & $n=\sqrt{2 / r-1}$ \\
Concentrator & $n=1 / r$ \\
\hline \hline
\end{tabular}

waves ${ }^{27}$ where the mapping with the electromagnetic waves is well supported.

Fig. 2(a) shows the variation of the refractive index along the direction of propagation for each one of the lenses described in Table I and Fig. 2(b) shows the corresponding solution for the radius-dependent plate thickness $h(r, \theta) / h_{b}$ obtained using Eq. (3). Notice that all the proposed flexural lenses present a refractive index larger than that of the background, therefore the thickness will always be smaller than that of the plate. In other words, all these lenses can be fabricated by gradually decreasing the plate's thickness.

The simulations have been performed using the one dimensional multilayer scattering method, described in the appendix of Ref. 24, where the continuous refractive index of each lens has been discretized in a number $N$ of concentric cylindrical layers. The vertical displacement $W_{n}$ in each layer satisfies the coordinate-independent flexural wave equation. Thus, the solution can be expanded as a linear combination of Bessel and Hankel functions (and their modified versions), as follows:

$$
\begin{aligned}
W_{n}= & \sum_{q}\left[A_{n, q}^{(1)} J_{q}\left(k_{n} r\right)+A_{n, q}^{(2)} I_{q}\left(k_{n} r\right)\right] e^{i q \theta} \\
& +\sum_{q}\left[B_{n, q}^{(1)} H_{q}\left(k_{n} r\right)+B_{n, q}^{(2)} K_{q}\left(k_{n} r\right)\right] e^{i q \theta},
\end{aligned}
$$

where $A_{n, q}^{(1)}, A_{n, q}^{(2)}, B_{n, q}^{(1)}, B_{n, q}^{(2)}$ are the coefficients of the expansion ( $A$ for the incoming wave and $B$ for the scattered one) and $k_{n}$ is the wave-number of the $n$-th layer. Boundary conditions at each layer are applied as described in Ref. 28, and

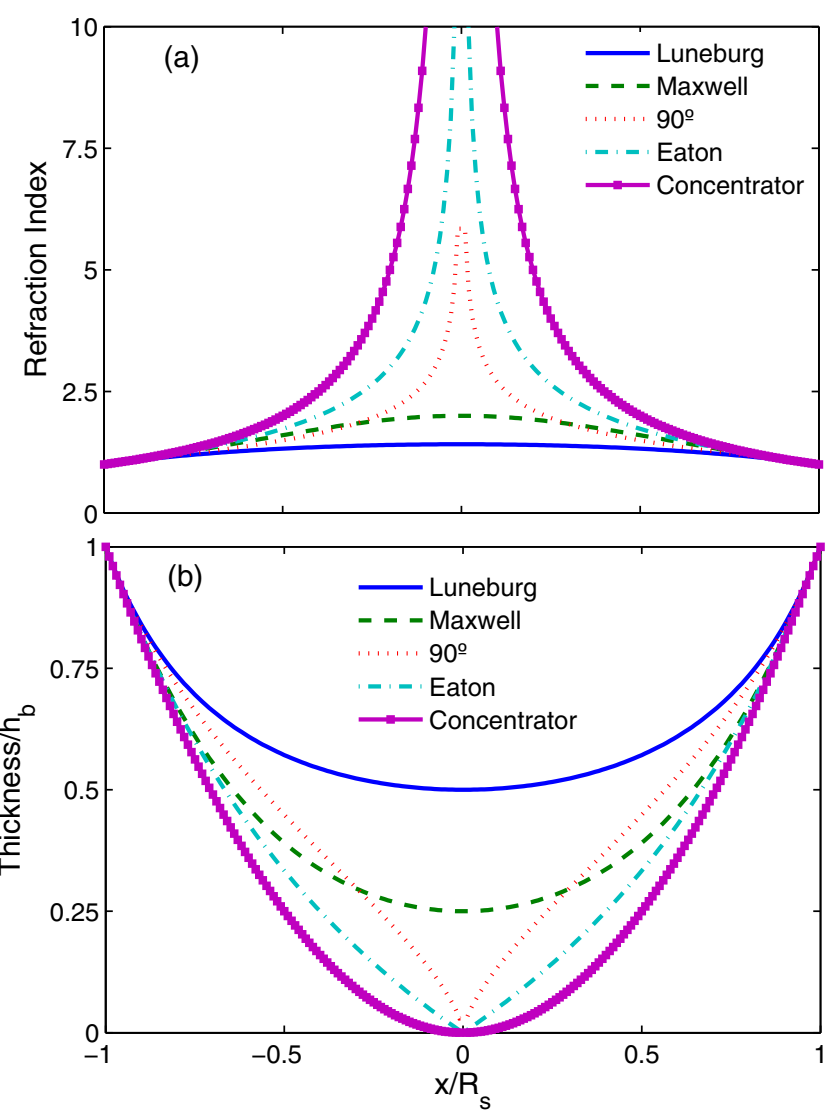

FIG. 2. Variation of the refractive index (a) and the thickness (b) of the studied lenses Vs the normalized x-coordinate. 

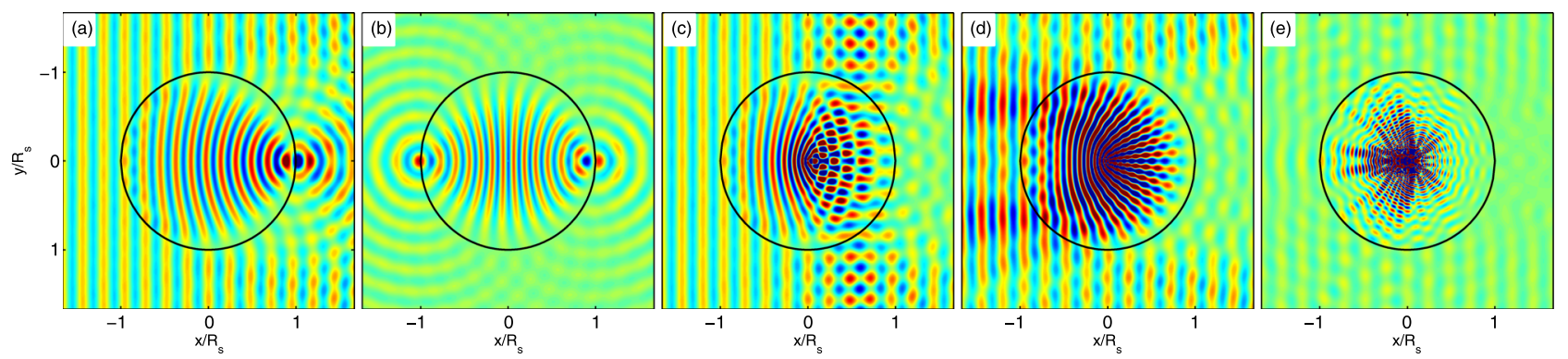

FIG. 3. Real part of the displacement $W(r, \theta)$ produced by a plane ((a), (c)-(e)) or cylindrical (b) wave impinging a Luneburg (a), Maxwell (b), $90^{\circ}$ rotating (c), Eaton (d), and concentrator (e) lens at $k R_{s}=8.3 \pi$. Color scale is the same as in Fig. 5.

then the coefficients of the expansion are determined in terms of the amplitude of the incident field.

A homogeneous infinite aluminum plate with $E=78.97$ (GPa), $\rho=2700\left(\mathrm{~kg} / \mathrm{m}^{3}\right)$, and $\nu=0.33$ has been considered for the simulations. The normalized thickness of the plate is $h_{b} / R_{s}=0.0167$. It is important to note in Fig. 2(a) that in some cases the thickness tends to zero as we approach the center of the lens. To avoid this singularity, a minimum thickness is established as $h_{\min }(r) / h_{b}=0.2 \times 10^{-3}$ which is small enough to be neglected. Each lens was discretized in $N=100$ layers. Our numerical simulations demonstrate that this discretization is a good approximation within the range of wavelengths used.

Fig. 3 shows the real part of the displacement $W(r, \theta)$ for the different type of lenses listed in Table I when a field with $k R_{s}=8.3 \pi\left(\lambda / R_{s}=0.24\right)$ is excited in the plate. Here, $\lambda=2 \pi / k$ and $k$ is obtained from the dispersion equation (2). Then, panel (a) shows a Luneburg Lens in which a plane wave is focused at the border of the device, panel (b) shows
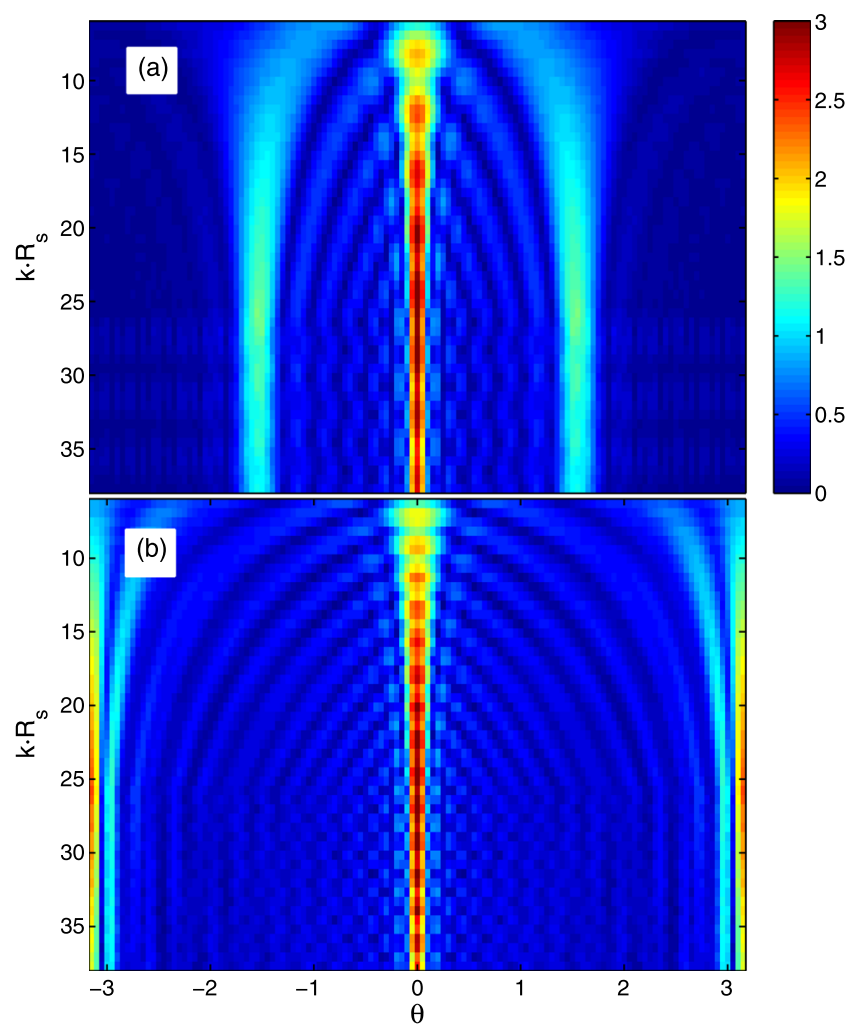

FIG. 4. Scattering cross-section produced by the $90^{\circ}$ rotating lens (a) and the Eaton lens (b). a Maxwell Fish-Eye, in this case a point source is excited at the border of the lens and it is focused at the opposite point. Panel (c) shows a $90^{\circ}$ rotating lens, where the incident plane wave is symmetrically redirected along the perpendicular direction of propagation, similarly as the Eaton lens shown in panel (d), where the wave is directed backwards this time. The concentrator shown in panel (e) is identical to the photonic black hole studied by Narimanov and Kildishev ${ }^{26}$ which was also tested for acoustic waves. ${ }^{27}$ As expected, the elastic counterpart exhibits similar behavior.
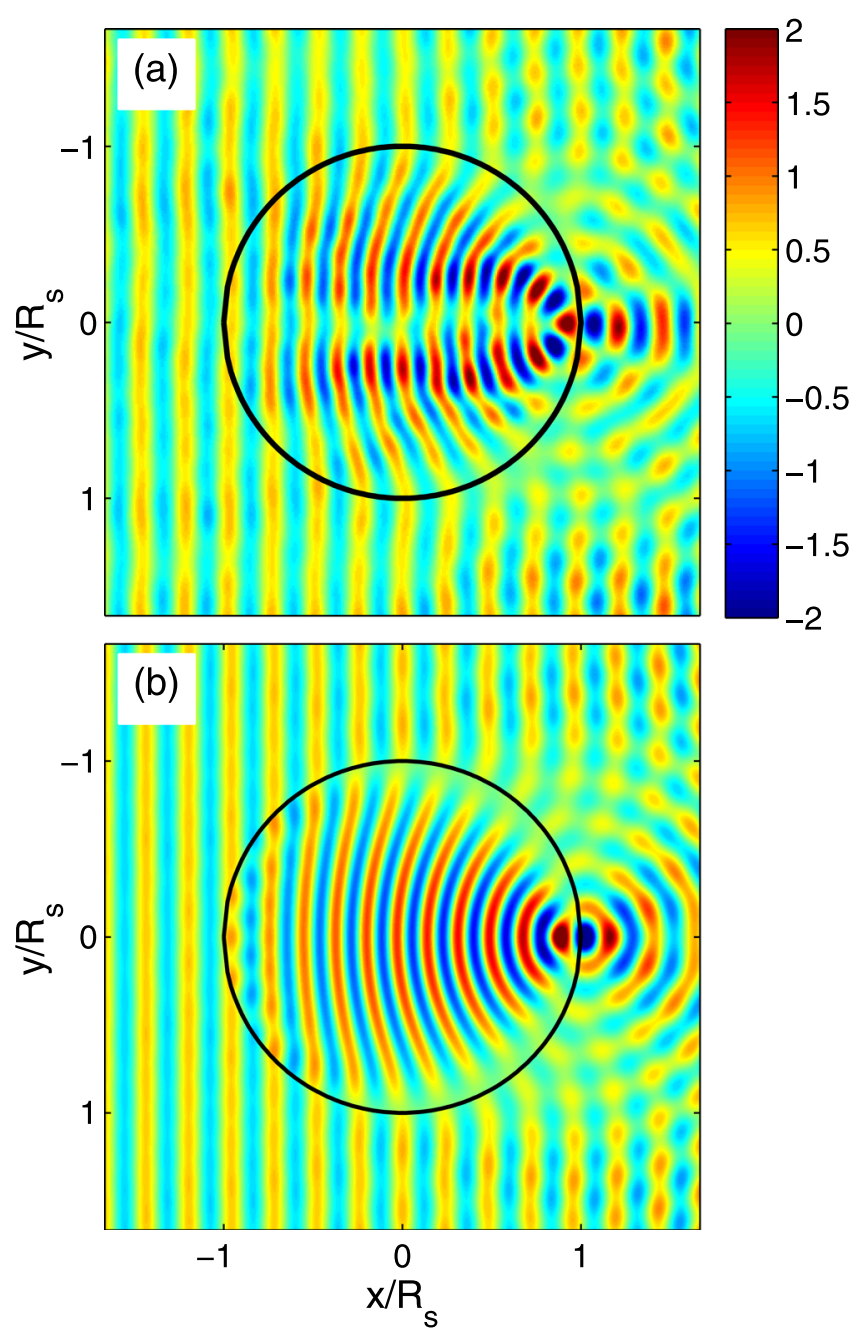

FIG. 5. Real part of the displacement $W(r, \theta)$ produced by a plane wave impinging a Luneburg lens. Simulations done with a commercial FEM simulator using the 3D elastic theory (a) and a the 1D multilayer simulator (b). 
As a parameter used to prove the broadband efficiency of the method to control the refractive index with the thickness, we have used the scattering cross section (SCS) defined at the far-field as

$$
\sigma(k, \theta) \equiv \lim _{r \rightarrow \infty}\left|\sqrt{r} W_{s c}(r, \theta)\right|,
$$

where $W_{s c}(r \theta)$ is the displacement of the scattered wave. The SCS has been calculated from $\theta \in[-\pi, \pi]$ and for a wide range of normalized wave-numbers $\left(k R_{s} \in[5,40]\right)$. Fig. 4(a), on one hand, shows $\sigma$ for the $90^{\circ}$ rotating lens. Note how it increases in the near of $\theta= \pm \pi / 2$ and becomes narrow when the normalized wave-number increases. By the other hand, Fig. 4(b) shows $\sigma$ for the Eaton lens. In this case, it increases in the near of $\theta= \pm \pi$ and also narrows with increasing normalized wave-number.

Finally, in order to test the flexural wave approximation, a numerical experiment employing the full 3D elastic equations, has been realized with a commercial finite element simulator. Fig. 5 shows the real part of the displacement $W(r, \theta, t)$ resulting when a plane wave impinges the Luneburg lens. Fig. 5(a) shows the results obtained from the commercial simulator and Fig. 5(b) the result given by our simulator based on the Timoshenko plate equation (1). Both results are very similar and show the expected behavior produced by a Luneburg Lens. Note that the result obtained by the commercial simulator is slightly distorted due to the difficulty of achieving zero reflectance on the boundary of the elastic plate. Moreover, it is a full 3D elastic simulation.

In summary, we have studied five different refractive lenses for flexural waves in thin plates. Their corresponding refractive index has been designed by adjusting locally the plate thickness. The results obtained confirm the expected behavior of the five lenses analyzed and support the refractive index model employed. It has also been demonstrated their broadband performance. We expect that our numerical demonstration of the reported flexural lenses will stimulate future experimental work looking for their practical realization.
This work has been supported by the U.S. Office of Naval Research under Grant No. N000140910554.

${ }^{1}$ A. Norris and C. Vemula, J. Sound Vib. 181, 115 (1995).

${ }^{2}$ V. Squire and T. Dixon, J. Sound Vib. 236, 367 (2000).

${ }^{3}$ A. Movchan, N. Movchan, and R. McPhedran, Proc. R. Soc. London, Ser. A 463, 2505 (2007).

${ }^{4}$ W.-M. Lee and J.-T. Chen, Int. J. Solids Struct. 47, 1118 (2010).

${ }^{5}$ W. Parnell and P. Martin, Wave Motion 48, 161 (2011).

${ }^{6}$ R. McPhedran, A. Movchan, and N. Movchan, Mech. Mater. 41, 356 (2009).

${ }^{7}$ J.-C. Hsu and T.-T. Wu, Phys. Rev. B 74, 144303 (2006).

${ }^{8}$ C.-Y. Huang, J.-H. Sun, and T.-T. Wu, Appl. Phys. Lett. 97, 031913 (2010).

${ }^{9}$ M. Farhat, S. Guenneau, and S. Enoch, Europhys. Lett. 91, 54003 (2010).

${ }^{10}$ T.-T. Wu, Y.-T. Chen, J.-H. Sun, S.-C. S. Lin, and T. J. Huang, Appl. Phys. Lett. 98, 171911 (2011).

${ }^{11}$ J. Pierre, O. Boyko, L. Belliard, J. Vasseur, and B. Bonello, Appl. Phys. Lett. 97, 121919 (2010).

${ }^{12}$ M. Farhat, S. Guenneau, S. Enoch, A. B. Movchan, and G. G. Petursson, Appl. Phys. Lett. 96, 081909 (2010).

${ }^{13}$ S. Bramhavar, C. Prada, A. A. Maznev, A. G. Every, T. B. Norris, and T. W. Murray, Phys. Rev. B 83, 014106 (2011).

${ }^{14}$ C. Wang and L. Rose, AIP Conf. 657, 1615 (2003).

${ }^{15}$ P. Fromme, P. D. Wilcox, M. J. Lowe, and P. Cawley, IEEE Trans. Ultrason. Ferroelectr. Freq. Control 53, 777 (2006).

${ }^{16}$ J. G. Berryman, J. Acoust. Soc. Am. 68, 1809 (1980).

${ }^{17}$ A. A. Krokhin, J. Arriaga, and L. N. Gumen, Phys. Rev. Lett. 91, 264302 (2003).

${ }^{18}$ D. Torrent, A. Håkansson, F. Cervera, and J. Sánchez-Dehesa, Phys. Rev. Lett. 96, 204302 (2006).

${ }^{19}$ N. Stenger, M. Wilhelm, and M. Wegener, Phys. Rev. Lett. 108, 014301 (2012).

${ }^{20}$ V. Krylov and F. Tilman, J. Sound Vib. 274, 605 (2004).

${ }^{21}$ D. O'Boy, V. V. Krylov, and V. Kralovic, J. Sound Vib. 329, 4672 (2010).

${ }^{22}$ V. V. Krylov and R. Winward, J. Sound Vib. 300, 43 (2007).

${ }^{23}$ V. Georgiev, J. Cuenca, F. Gautier, L. Simon, and V. Krylov, J. Sound Vib. 330, 2497 (2011).

${ }^{24}$ A. Climente, D. Torrent, and J. Sánchez-Dehesa, J. Appl. Phys. 114, 214903 (2013).

${ }^{25}$ M. Šarbort and T. Tyc, J. Opt. 14, 075705 (2012).

${ }^{26}$ E. Narimanov and A. Kildishev, Appl. Phys. Lett. 95, 041106 (2009).

${ }^{27}$ A. Climente, D. Torrent, and J. Sánchez-Dehesa, Appl. Phys. Lett. 100, 144103 (2012).

${ }^{28}$ S. Timoshenko, Theory of Plates and Shells (McGraw-Hill, 1940). 